\title{
Design on Cardiovascular System Indicators Monitoring Software for Undergraduate
}

\author{
Guoquan Zhang \\ Department of Physical Education, Bohai University, Jinzhou, 121013, China \\ 673722120@qq.com
}

Keywords: undergraduate; cardiovascular system; indicators monitoring; software design

\begin{abstract}
The cardiovascular system is a closed pipe system, which is composed of the heart and blood vessels. It can ensure the relative constant and the metabolism of the body's environment. The diagnosis and monitoring of cardiovascular system is the key part of the sports medical supervision, which plays an important role in undergraduate physical function assessment and training monitoring. In this paper, we study the problem of the cardiovascular system of undergraduate based on manual method. Firstly, the heart rate, blood pressure and electrocardiogram of the cardiovascular system function evaluation are classified into 12 categories; Then, the research is composed of layer database, layer component, layer and presentation layer business and other four layers of the new C/S software architecture; Finally, research ADO data access technology on connection, command and recordset. The research content of this paper has solved the key technology of the software development, which has laid a foundation for the development and implementation of the software.
\end{abstract}

\section{Introduction}

The cardiovascular system is a closed pipe system, which is composed of the heart and blood vessels. Heart is the power organ. Blood vessel is the pipeline. The heart has the rhythm of contraction and relaxation, and promote blood circulation in blood vessels in a certain direction, called the blood circulation. Blood circulation is one of the most important physiological functions of the organism. Because the blood circulation, all functions of the blood was able to achieve, and adjust the blood distribution, need to adapt to the activities of the organs and tissues, so as to ensure the normal environment of the body relatively constant and metabolism. Once the cycle stops, the life activity can't be carried out normally, and the final result will lead to the death of the body [1].

In order to implement the guiding ideology of "health first", strengthen the school physical education, promote students to actively participate in physical exercise, develop good exercise habits, improve the health level, the Ministry of Education promulgated the "students physical health standards", and clearly defined the health of students' physical health evaluation index, score table, the implementation [2].In promoting the health of undergraduate, good heart pump blood function is particularly important. Therefore, the diagnosis and monitoring of the structure and function of the cardiovascular system is the key part of the sports medical supervision.

\section{Monitoring Indicators}

The common indicators of cardiovascular system function were heart rate, blood pressure, electrocardiogram and so on. Heart rate is the frequency of the mechanical activity of the heart, that is, the number of beats per minute [3]. Heart rate can be expressed by the pulse, the commonly used heart rate are the basis of heart rate, quiet rate, exercise rate and heart rate after exercise.Arterial blood pressure of the human body is relatively stable. If blood pressure is too low, reduced blood supply can not meet the metabolic needs of the body tissue. The tissue may be due to ischemia, hypoxia and cause disease. If blood pressure is too high, the resistance of the ventricular ejection is too large, the load of the myocardium will grow. The change of blood pressure in training was related to the intensity of exercise, and the increase of systolic blood pressure and diastolic blood pressure decreased significantly after training.After training, the systolic blood pressure was 
significantly increased, diastolic blood pressure also increased, or blood pressure response and intensity stimulation, recovery time, and so on, the function is not good. The heart electrical change curve recorded in a certain part of the body or body is called an electrocardiogram.

Undergraduates cardiovascular system needs to monitor 12 categories of indicators, namely vital capacity, suffocating time, stroke index, cardiac output, cardiac index, VPE, VPE Index, effective utilization rate of left ventricular myocardial energy, echocardiography, heart rate, blood pressure, electrocardiogram. Some indicators include sub categories, some indicators do not. Among them, the "echocardiography" index contains 10 sub categories, namely end-diastolic left ventricular posterior wall thickness, end-systolic left ventricular posterior wall thickness, left ventricular end-diastolic diameter, left ventricular end systolic diameter, end-systolic thickness of interventricular septum, end-diastolic interventricular septum thickness, small-axis shortening rate of left ventricular, left ventricular mass, ejection fraction, stroke volume. The "rate heart" contains 4 sub categories, namely the basis of heart rate, resting heart rate, exercise heart rate, post-exercise heart rate. The "pressure blood" contains 6 sub categories, namely systolic blood pressure, diastolic blood pressure, pulse pressure, arterial pressure, aortic emptying coefficient, total peripheral resistance. Indicators include "Class, Subclass, Indicators Name and Measuring Unit", and indicator information for cardiovascular system monitoring in undergraduates is shown in Table 1 [4].

Table 1. Monitoring indicators on cardiovascular system

\begin{tabular}{|c|c|c|c|}
\hline Class & Subclass & Indicators Name & $\begin{array}{c}\text { Measuring } \\
\text { Unit }\end{array}$ \\
\hline 0201 & & vital capacity & $\mathrm{L}$ \\
\hline 0202 & & suffocating time & $\mathrm{sec}$ \\
\hline 0203 & & stroke index & $\mathrm{ml}$ \\
\hline 0204 & & cardiac output & $\mathrm{L}$ \\
\hline 0205 & & cardiac index & $\mathrm{L}$ \\
\hline 0206 & & VPE & $\mathrm{kg} / \mathrm{b}$ \\
\hline 0207 & & VPE Index & kg.min.m ${ }^{2}$ \\
\hline 0208 & & effective utilization rate of left ventricular myocardial energy & $\%$ \\
\hline \multirow[t]{11}{*}{0209} & & echocardiography & \\
\hline & 021001 & end-diastolic left ventricular posterior wall thickness & $\mathrm{mm}$ \\
\hline & 021002 & end-systolic left ventricular posterior wall thickness & $\mathrm{mm}$ \\
\hline & 021003 & left ventricular end-diastolic diameter & $\mathrm{mm}$ \\
\hline & 021004 & left ventricular end systolic diameter & $\mathrm{mm}$ \\
\hline & 021005 & end-systolic thickness of interventricular septum & $\mathrm{mm}$ \\
\hline & 021006 & end-diastolic interventricular septum thickness & $\mathrm{mm}$ \\
\hline & 021007 & small-axis shortening rate of left ventricular & $\%$ \\
\hline & 021008 & left ventricular mass & g \\
\hline & 021009 & ejection fraction & $\%$ \\
\hline & 021010 & stroke volume & $\mathrm{ml} / \mathrm{b}$ \\
\hline \multirow[t]{5}{*}{0210} & & heart rate & beats / min \\
\hline & 021101 & the basis of heart rate & beats / $\min$ \\
\hline & 021102 & resting heart rate & beats / $\min$ \\
\hline & 021103 & exercise heart rate & beats / min \\
\hline & 021104 & post-exercise heart rate & beats / $\min$ \\
\hline
\end{tabular}


Table 1. Monitoring indicators on cardiovascular system

\begin{tabular}{|c|c|c|c|}
\hline Class & Subclass & Indicators Name & $\begin{array}{c}\text { Measuring } \\
\text { Unit }\end{array}$ \\
\hline \multirow[t]{7}{*}{0211} & & blood pressure & $\mathrm{mmHg}$ \\
\hline & 021201 & systolic blood pressure & $\mathrm{mmHg}$ \\
\hline & 021202 & diastolic blood pressure & $\mathrm{mmHg}$ \\
\hline & 021203 & pulse pressure & $\mathrm{mmHg}$ \\
\hline & 021204 & arterial pressure & $\mathrm{mmHg}$ \\
\hline & 021205 & aortic emptying coefficient & \\
\hline & 021206 & total peripheral resistance & $\mathrm{sec} / \mathrm{cm}^{-1}$ \\
\hline 0212 & & electrocardiogram & \\
\hline
\end{tabular}

\section{Software Architecture}

System structure design refers to the hardware system structure, with the software system and other supporting equipment design. The hardware system includes the configuration of basic components and the structure of network communication. Software systems usually adopt distributed structure, which means that the use of communication facilities will be a number of actually independent, in different regions of the computer into a computer can communicate with each other, sharing resources, mutual coordination, with the operation of the system.From the eighty's, this system has a considerable development, and more and more for the vast number of users are willing to accept, to the ninety's, into the PC machine, workstations as the representative of the environment. This new distributed environment is the core of the $\mathrm{C} / \mathrm{S}$ architecture. $\mathrm{C} / \mathrm{S}$ software architecture is shown in Fig. 1.

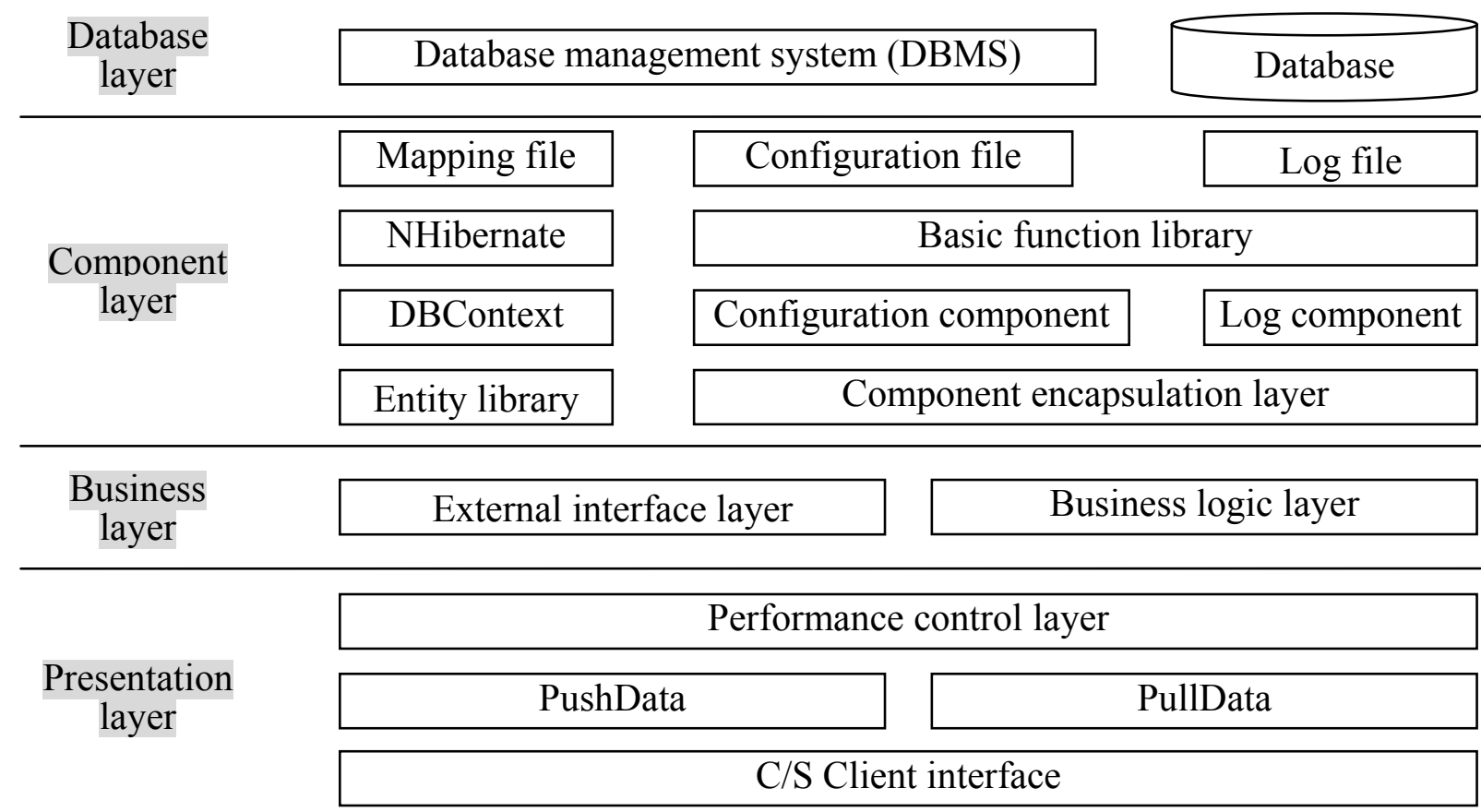

Fig. 1. C/S Software architecture

$\mathrm{C} / \mathrm{S}$ (Client/Server) structure, which take advantage of the hardware environment of the two ends. The task is assigned to the Client and Server. The communication overhead is reduced. At present, most of the application software system is the two layer structure of Client/Server. Because the software application system is developing in the distributed application system, Web and Client/Server application can be used in the same business process and application of different 
modules to shared logic components. Therefore, both internal and external users can access the application system, through the existing application system logic can be extended to a new application system. $\mathrm{C} / \mathrm{S}$ structure of the advantages of the software in the following two aspects $[5,6]:$

(1) Application server running data load is light. The most simple $\mathrm{C} / \mathrm{S}$ architecture of the database application is composed of two parts, that is, the client application and database server program. A machine running a database server program, also known as an application server. Once the server program is started, it is ready to respond to the client's request. When any operation is required, the client program automatically seeks the server program. And to its request, the server program according to the predetermined rules to make a response, back to the results. The application server running data load is lighter.

(2) Data storage management functions are more transparent. In the application of database, the function of data storage management is carried out by the server program and the client application. For end users working in front of the procedure, need not intervene process behind, you can complete all the work. In the application of the client server architecture, the main data operations are delivered to the server and the network. In the C/S system, the database can not truly become public, professional warehouse. It needs special management.

\section{Data Access Technology}

ADO (Data Objects ActiveX) is Microsoft's latest data access technology. It is a former DAO, especially RDO data access interface of the successor product. ADO access data source is achieved by accessing the OLEDB data and providing the program. The use of a large number of COM interfaces in the DB OLE program is a high-level access technology. While inheriting the advantages of DB OLE, it simplifies the operation of DB OLE. In addition, the ADO technology can be used in the form of ActiveX control to simplify programming, ADO based on COM and the application of the ADO generated by the application of memory. Therefore, ADO technology has become the development direction of data access technology. The ADO object model is shown in Fig. 2.

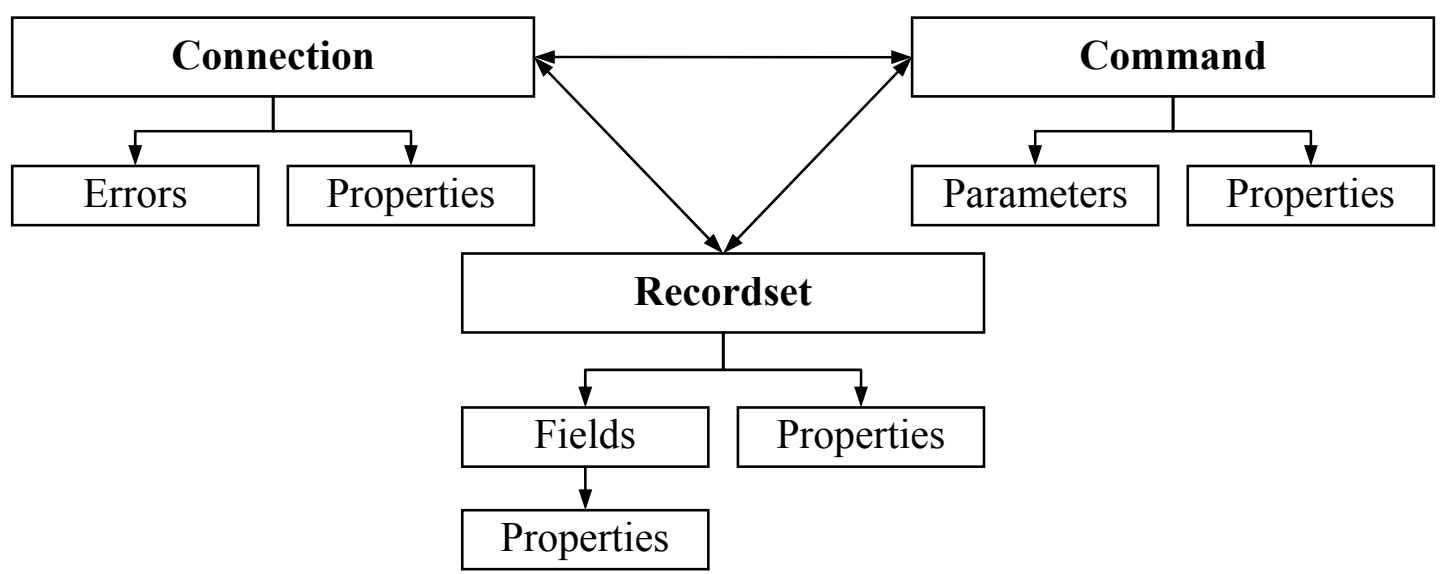

Fig. 2. ADO object model

In the ADO model, the main objects include 3, Command, Recordset, Errors, etc., the other 4 sets of objects Properties, Parameters, Fields and Error, respectively, corresponding to Property, Parameter, Connection and Field objects. Command is an optional object, which depends on whether the DB OLE data provider implements the Command interface. Since DB OLE can provide a relational data source can also provide a non relational data source, so the use of the traditional SQL command query data on non relational data sources may be invalid, and even command objects can not be used [7,8].

(1) Connection object. Connection object represents a connection between the data source, the Connection ADO object, package the DB OLE's data source object and session object. According to 
the different performance of DB OLE provider. the characteristics of Connection object are also different. So the method and property of Connection object are not always available. Connection object is one of the basic objects of ADO, independent of all other objects.

(2) Command object. Command object represents a command, you can perform the relevant operations of the data source through its method, such as query, modify, etc.. When the Command object is executed, the Connection object can be specified by the ActiveConnection property. It can also be independent from the Connection object. The connection string is directly specified, even if the connection string is the same as the Connection object.

(3) Recordset object. The Recordset object represents the result of a table's record set or a command to execute, and always has a current record in the record set. The record collection is the basic object of the ADO management data, and all the Recordset objects are managed by a table like structure in which each row is a record, and each column corresponds to a domain. The Recordset object is accessed through a cursor.

\section{Conclusion}

The cardiovascular system is composed of the heart, arteries, capillaries and veins. The heart is the key link between arteries and veins. It is the "power pump" of the cardiovascular system, and has important endocrine function. The heart has a rhythm to the contraction and relaxation, the blood flow is not stopped by the arterial injection, by intravenous inhalation, to ensure blood flow in the heart of the continuous flow of continuous flow. Arteries are blood vessels of the blood vessels, which is the blood vessel that leads the blood to the heart, and the capillary is the conduit between the arteries and veins. It is an important work to improve the health quality of college students. The research content of this paper is to solve the problem of the key technology of the software development, which lays the foundation for the development and implementation of the software.

\section{References}

[1]

$$
\text { Baidu Encyclopedia, "Cardiovascular system," }
$$

http://www.baidu.com/s?wd=\%D0\%C4\%D1\%AA\%B9\%DC\%CF\%B5\%CD\%B3\&tn=synacast ___adr, 2015-9-11.

[2] X. Dai, L. Zhu, H. G. Xie, "Reflection and Optimization on Evaluation Efficiency of National Student Physical Health Standard :Construction on Physical Fitness Early Warning System of College Students," China Sport Science and Technology, vol. 48, no. 9, pp. 75-82, 2012.

[3] Road passenger Baba online document sharing platform, "Evaluation index and method of performance of athletes' body system," http://www.doc88.com/p-2723098578855.html, 2015-9-11.

[4] General Administration of Sport of China, "Requirements of athletes' physical morphometrics and functions digital archives," Sports industry standard of the people's Republic of China, 2014-5-22.

[5] C. L. Liu, "Development and application of C/S and B/S hybrid architecture," Electronic Design Engineering, vol. 23, no. 14, pp. 26-28, 2015.

[6] HE Xiaoyan, CHEN Bing, WANG Hui, et al, "Component Management System Based on C/S Model," Journal of Wuhan University of Technology(Information \& Management Engineering), vol. 37, no. 2, pp. 143-146, 2015.

[7] J. Chen, "Research and implementation of database access based on ADO Technology," Technology Wind, vol. 27, no. 12, pp. 51, 2014.

[8] Tang Jiao, Song Maozhong, "Crane safety monitoring application of database access based on ADO," Microcomputer \& Its Applications, vol. 33, no. 19, pp. 102-104, 2014. 